\title{
Conséquences des anomalies de la structure chromosomique chez les animaux domestiques
}

\author{
CP Popescu
}

INRA, CRJ, Laboratoire de Cytogénétique, 78350 Jouy-en-Josas, France

(28e Réunion de la Société Française pour l'Étude de la Fertilité; Paris, 19-21 octobre 1989)

Résumé - Les anomalies de la structure chromosomique n'ont généralement pas d'effet phénotypi-
que visible, mais sont souvent associées à des troubles de la reproduction. Chez le bœeuf, le mouton
et la chèvre, la translocation de type robertsonien est l'anomalie de structure la plus fréquente. En
revanche, chez le porc les translocations réciproques semblent les plus répandues. Chez les bovins
et le porc, il a été possible de recueillir une information assez complète concernant la fréquence de
ces anomalies et leurs effets sur les performances de reproduction, dont on peut ensuite calculer les
conséquences économiques. Ainsi chez les bovins les effets défavorables de la fusion centrique
$1 / 29$ ont justifié l'instauration d'une politique d'éradication, basée sur l'élimination des taureaux por-
teurs dans les centres d'insémination artificielle. Chez le porc, les translocations réciproques rédui-
sent parfois de $50 \%$ la taille des portées et provoquent ainsi des pertes économiques considérables.

chromosome / anomalie / bovins / porcins / fertilité

Summary - Consequences of structural chromosomal abnormalities in farm animals. Abnormalities in chromosome structure generally have no phenotypic expression but are very often associated with reproductive disorders. In cattle, sheep and goats, the robertsonian translocation seems to be the most frequent abnormality of chromosome structure. In the pig, reciprocal translocations are very common. The accumulation of data on the frequency of such abnormalities and their effects on reproductive performance prompted an evaluation of their economic consequences in cattle and pigs. In cattle, because of the negative effect of 1/29 trans/ocation, an eradication program, based on the removal of carrier bulls from artificial insemination centers was established. In pig, the main effect of the reciprocal translocations was a reduction in the number of offspring, up to $50 \%$, thus representing a considerable economic loss.

chromosome / abnormality / cattle / pig / fertility

\section{INTRODUCTION}

Les échecs de la reproduction, regroupés sous le terme général d'infertilité, expriment l'incapacité d'un organisme à engendrer des descendants ou une réduction notable du nombre de ses descendants, par rapport à la moyenne de la population. Les causes d'infertilité sont multiples : infec- tieuses, nutritionnelles ou génétiques. Parmi ces dernières, les anomalies chromosomiques sont responsables, chez l'homme d'environ $50 \%$ des avortements spontanés précoces (Boué et Boué, 1975). Les anomalies de nombre survenues au cours de la gamétogénèse ou au moment de la fécondation sont souvent la cause de ces échecs de la reproduction. 
En raison de leur caractère accidentel, les études prospectives de ces anomalies numériques restent limitées. Les parents porteurs d'une anomalie de la structure chromosomique constituent aussi un groupe avec un risque très élevé d'échecs de la reproduction. Le mode de ségrégation méiotique et les différents types de caryotypes déséquilibrés en résultant peuvent être étudiés soit dans les configurations des spermatocytes au stade pachytène, soit dans les produits d'avortements spontanés. Le développement récent des techniques de fécondation de l'ovocyte de hamster par des spermatozoïdes humains permet d'identifier les caryotypes anormaux dans le pronucléus mâle. Ainsi, à ce jour, le mode de ségrégation méiotique d'une dizaine d'anomalies, en particulier des translocations réciproques et robertsoniennes, a été analysé dans les pronucléus humains.

\section{LES ANOMALIES DE LA STRUCTURE CHROMOSOMIQUE}

Les anomalies de la structure chromosomique apparaissent généralement après une cassure d'un bras chromosomique au stade préméiotique ou méiotique. Le fragment de chromosome résultant d'une cassure peut être perdu (déficience ou délétion), subir une rotation (inversion) ou être transloqué sur un autre chromosome.

Certaines anomalies de la structure perturbent le déroulement normal de la méiose. Ainsi les inversions provoquent la formation d'une boucle qui ramène dans le bivalent méiotique, les segments inversés face à face avec le segment homologue du chromosome normal. La formation des chiasmas à l'intérieur de la boucle peut produire des cassures. Les gamètes qui en résultent peuvent être déséquilibrés par défaut ou par excès d'un segment chromosomique.

Les translocations réciproques résultent d'un échange de segments entre deux chromosomes non homologues. L'anomalie peut impliquer n'importe quel chromosome du complément et les points de cassure varient d'une translocation à une autre. Le sujet porteur hétérozygote à l'état équilibré présente un phénotype tout à fait normal.

A la méiose, les deux chromosomes remaniés s'associent avec leurs homologues normaux pour former le quadrivalent. Après la ségrégation à l'anaphase, il en résulte 3 types de gamètes : normaux, porteurs de l'anomalie mais à l'état équilibré et des gamètes déséquilibrés par excès ou par défaut. L'analyse de la configuration du quadrivalent peut avoir une certaine valeur prédictive sur le mode de ségrégation des chromosomes et les types de gamètes qui en résultent.

Les gamètes déséquilibrés, selon la taille des segments transloqués et de leur contenu génique, peuvent donner des embryons viables ou létaux.

Les translocations de type robertsonien, ou fusion centrique, représentent un cas particulier de translocation réciproque dans lequel les deux chromosomes impliqués sont des acrocentriques. Les chromosomes fusionnent à la suite des deux cassures produites respectivement au-delà et en deçà du centromère. II en résulte un nouveau chromosome méta- ou submétacentrique et une réduction du nombre de chromosomes d'une unité. A la méiose, la configuration typique des chromosomes impliqués dans la translocation robertsonienne est le trivalent. La non disjonction de l'un ou l'autre des 2 chromosomes libres qui constituent le trivalent provoque la formation de gamètes déséquilibrés par excès ou par défaut d'un chromosome en- 
tier (monosomique ou disomique). Après fécondation, les embryons déséquilibrés seront monosomiques ou trisomiques, pour l'un ou l'autre des deux chromosomes fusionnés.

\section{LES ANOMALIES \\ DE STRUCTURE CHROMOSOMIQUE CHEZ LES ANIMAUX DOMESTIQUES}

On estime que dans l'espèce humaine $0,75 \%$ des nouveau-nés et $5 \%$ des mortnés portent une anomalie chromosomique. A la conception, chez l'homme, la fréquence des anomalies chromosomiques se situerait aux environs de $20 \%$ (Chandley, 1981). L'espèce humaine serait ainsi, parmi les espèces de mammifères étudiées, celle qui possède la fréquence la plus élevée d'anomalies chromosomiques.

Chez les animaux domestiques, les données sur la fréquence des anomalies chromosomiques varient selon l'espèce et les conditions d'élevage. D'une manière générale on remarque que les anomalies autosomiques de nombre sont très peu fréquentes. Au contraire, certaines anomalies de la structure chromosomique présentent dans certaines espèces d'élevage des fréquences très élevées.

\section{Les bovins}

Le bœuf domestique est probablement, après l'homme et la souris, l'espèce de mammifères la plus étudiée du point de vue cytogénétique. Ceci s'explique par la découverte en 1964 en Suède, d'une anomalie de structure (fig 1) la translocation robertsonienne 1/29 (Gustavsson et Rockborn, 1964) qui a des conséquences néfastes pour l'élevage. En effet, elle réduit la fertilité des femelles porteuses hétérozygotes d'environ $10 \%$. Chez les mâles hété- rozygotes, Gustavsson (1969) a observé une libido et des caractéristiques spermatiques, tels la mobilité et le volume normaux.

Depuis l'observation princeps de Gustavsson et Rockborn (1964) en Suède, la translocation $1 / 29$ a été retrouvée dans une cinquantaine de races bovines répandues sur les cinq continents.

La fréquence des porteurs hétérozygotes de cette anomalie est très variable selon la race et la taille de l'échantillon étudié. Dans certaines races à très petit effectif, telle la British White (Eldridge, 1975) ou la race Corse (Hari et al, 1984) elle atteint des fréquences très élevées, respectivement de 60 et $40 \%$.

Une étude ayant pour but d'estimer la fréquence de cette anomalie en race Blonde d'Aquitaine, a été récemment effectuée en France. Sur un échantillon représentatif de plus de 2000 génisses, la fréquence des hétérozygotes a été estiméo à $14,2 \%$ (Frebling et al, 1987). L'étude a également montré que parmi différents facteurs pris en considération, la région et le mode de reproduction, insémination artificielle ou monte naturelle, jouent un rôle important dans les variations de la fréquence de l'anomalie.

La réduction de la fertilité des animaux hétérozygotes pour cette anomalie a été expliquée par Gustavsson (1969) par une augmentation de la mortalité embryonnaire. II a proposé un schéma de la méiose chez un hétérozygote pour cette translocation et des zygotes qui en résultent après la fécondation par un gamète normal (fig 2). L'on remarque sur ce schéma la formation de plusieurs types de gamètes déséquilibrés par excès ou par défaut d'un chromosome. Ces gamètes, qui gardent leur capacité de fécondation normale, donnent après la fécondation des embryons non viables, monosomiques ou 
$\boldsymbol{\omega}$

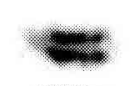

(2.)

ำ

$\infty$

$\underline{-}$

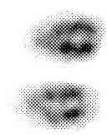

$\pm$

고.

$\times$

눌료

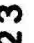

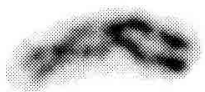

N

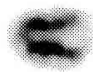

in

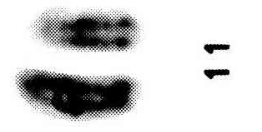

23

2

$\mathcal{2}$

N

$\stackrel{2}{\sim}$

…

I

$\stackrel{9}{N}$

$\dddot{0}$

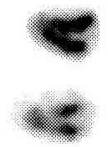

N

2.)

2.2

2.2

$\underset{N}{\infty}$

2.).

․․

2

$\bar{N}$

2.

a

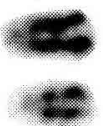

$\sqrt{2}$

ㄱ․․

$\hat{n}$

$\stackrel{\bullet}{\sim}$
오ำ

요 
trisomiques, pour l'un ou l'autre des deux chromosomes impliqués dans l'anomalie.

Ce schéma théorique proposé par Gustavsson a été validé par des études faites sur des cellules méiotiques et sur des embryons. Plusieurs études faites sur les chromoscmes méiotiques des taureaux hétérozygotes (fig 3) ont mis en évidence une certaine proportion de gamètes déséquilibrés (revue Popescu, 1989). La mise en évidence des caryotypes monosomiques pour le chromosome 1 chez des embryons à j 13 issus d'un père hétérozygote (Popescu, 1980) (fig 4) et des trisomiques pour le même chromosome chez des embryons à j 7 (King et al, 1980) a confirmé la validité de ce schéma et accrédité le rôle de la mortalité embryonnaire dans la réduction de la fertilité des hétérozygotes. Par ailleurs, on n'a jamais trouvé chez les veaux nouveau-nés ou adultes de trisomie ou monosomie pour les 2 chromosomes impliqués dans cette anomalie.

En Suède, le ministère de l'agriculture avait estimé qu'en raison de la réduction de la fertilité provoquée par cette anomalie, les éleveurs subissaient une perte d'environ $2000000 \mathrm{~F}$ par an. L'anomalie affectait à la fin des années soixante $14 \%$ de l'effectif de la race Pie Rouge Suédoise. Les Suédois ont alors décidé l'élimination de tous les taureaux IA porteurs de l'anomalie et l'examen des jeunes taurillons, avant la mise en testage. La mise en place d'une politique d'éradication a eu lieu dans beaucoup de pays d'Europe ainsi qu'un contrôle cytogénétique des animaux importés.

Sur un plan plus général, la découverte de cette anomalie a eu un impact considérable sur la cytogénétique des animaux domestiques. C'est pour la recherche de cette anomalie que d'énormes populations d'animaux et en particulier des reproducteurs, ont été examinés du point de vue cytogénétique.

Elle a également contribué à développer l'idée du danger que représentent des anomalies chromosomiques, qui se propagent d'une manière insidieuse, sans manifestations phénotypiques et qui provoquent des pertes économiques considérables.

Les translocations robertsoniennes sont de loin, les anomalies de structure les plus répandues chez l'espèce bovine, car plus d'une vingtaine de types différents ont été décrits. D'autres anomalies de la structure chromosomique sont citées dans la littérature, toujours associées à une réduction de la fertilité (Popescu, 1989).

\section{Les ovins et les caprins}

Chez le mouton, 3 translocations Massey I, II, III de type robertsonien ont été mises en évidence (Bruere, 1974) en NouvelleZélande. Trois races semblent être les plus affectées : Romney, Drysdale et Perendale (Bruere et al, 1976). Dans Romney, l'une des trois anomalies affecte $26 \%$ de l'effectif. Les travaux de Bruere et al (1976) sur les chromosomes méiotiques des mâles hétérozygotes ont mis en évidence l'existence des gamètes à caryotype déséquilibré. Bruere (1974) affirme qu'en comparant les taux de mise bas des femelles hétérozygotes avec la moyenne nationale de la race il ne trouve pas de différence significative. Ceci s'expliquerait par la dégénérescence de spermatocytes à caryotype déséquilibré. Cette sélection gamétique est contestée par plusieurs auteurs (Popescu, 1989).

Chez la chèvre, quelques cas de fusions centriques ont été signalés, dont un

Fig 1. Caryotype d'une cellule de vache homozygote pour la translocation 1/29. 
cellule diploide $\left.1 / 29\} \oint_{29}\right\}_{1}$

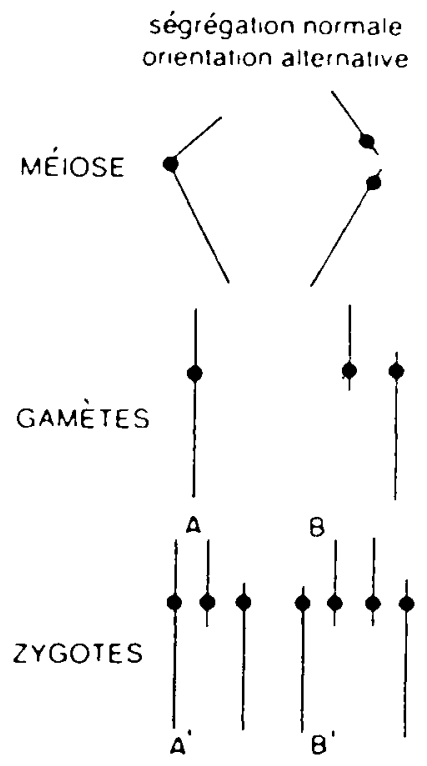

A équilibre, fusion centrique

$B$ équitibre normal

C disomique 29

D nullisomique 29

E disomique ।

F nulisomique I ségrégatıon anormale orientation adjacente
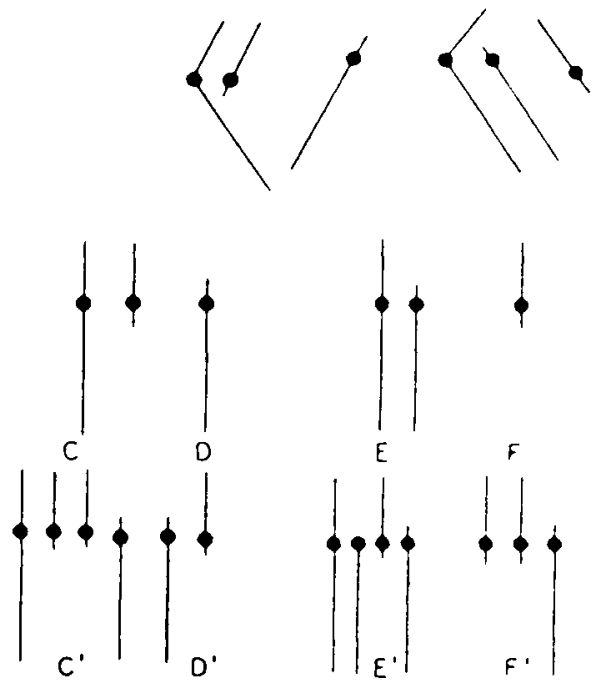

$$
\phi
$$

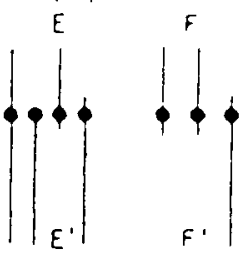
A équilibre fusion centrique
8. équilibre normal
C. insomique 29
D monosomique 29
E trisomique I
$F$ monosomique I

Fig 2. Les différents types de gamètes et zygotes produits par un bovin hétérozygote pour la translocation 1/29 (d'après Gustavsson, 1969).

Fig 3. Caryotype d'une cellule méiotique au stade de diacinèse d'un taureau hétérozygote pour la translocation $1 / 29$. 
$\omega$

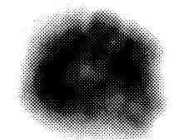

N

เ

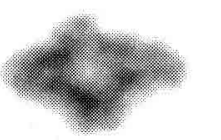

$=$

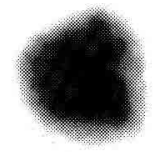

$=$

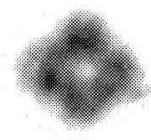

N

N

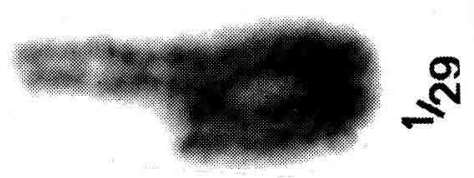

$\infty$ N $m$

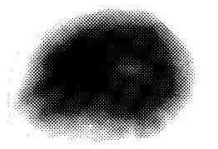

$\infty$

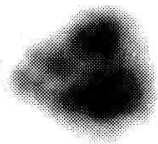

ำ

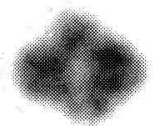

$\bar{N}$

우

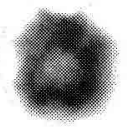

$\stackrel{0}{N}$ 
$\infty$

v

$\infty$

2.

.

$=$
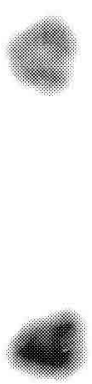

?

ㄱ.

$m$

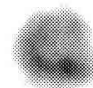

$\sigma$
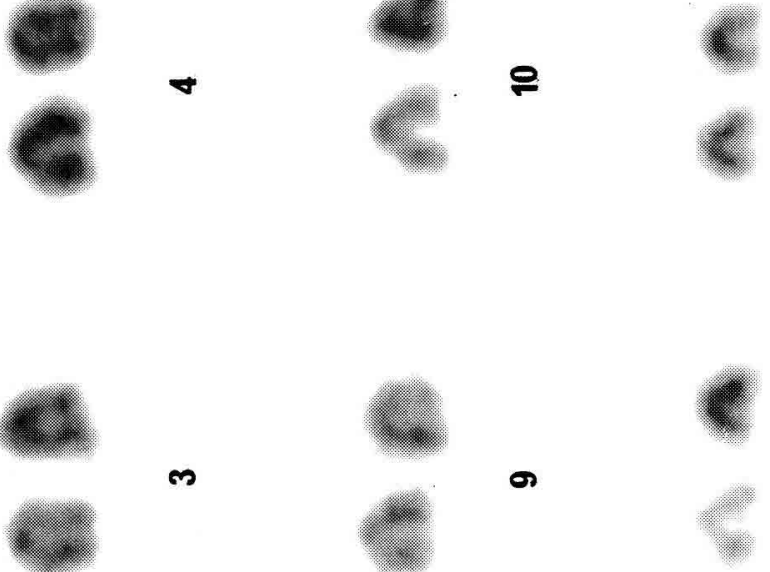

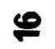

$\approx$

2.

N

28

ลี

2.

ส

2.

오

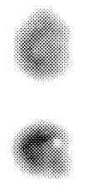

E

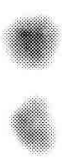

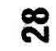

ล

ำ

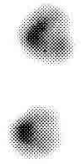

ลิ

N

$\infty$
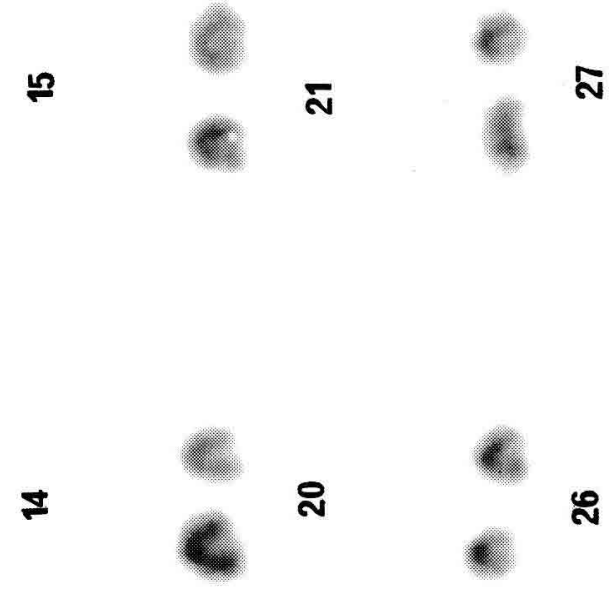

2.

르

$m$

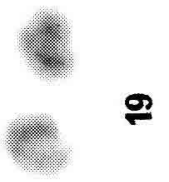


en France (Popescu, 1972) ayant le même effet négatif sur la fertilité que la translocation 1/29 chez les bovins (Ricordeau, 1972).

\section{Les porcins}

Contrairement aux autres espèces d'élevage qui possèdent beaucoup de chromosomes dont la plupart sont acrocentriques, le porc a 38 chromosomes de morphologie différente, ce qui facilite leur étude.

Les anomalies de structure les plus fréquentes connues chez cette espèce sont les translocations réciproques : plus d'une trentaine de types différents, sont décrits dans la littérature. Leurs conséquences pour l'élevage sont plus graves que celles des translocations robertsoniennes, car la réduction de la fertilité est beaucoup plus importante, de $25 \%$ jusqu'à la stérilité totale (Popescu et Legault, 1988).

En France, le programme national de gestion technique de troupeaux de truies classe les verrats en fonction de la taille des portées qu'ils ont conçues. II permet en conséquence une détection rapide des verrats hypoprolifiques. Depuis une dizaine d'années, le laboratoire de cytogénétique de l'INRA de Jouy étudie systématiquement les verrats hypoprolifiques ayant produit en moyenne 8 porcelets par portées ou moins, sur au moins 6 portées (Popescu et Legault, 1988). Dans le lot des verrats hypoprolifiques, 6 translocations réciproques différentes ont été identifiées, l'une d'entre elles ayant été observée 6 fois. La réduction de la taille des portées se situe entre 26 et $49 \%$ (Popescu et Legault, 1988) (figs 5 et 6 ).
Chez le porc, la formation de gamètes à caryotype déséquilibré a été vérifiée par Akesson et Henricson (1972) pour la translocation 11/15, King et al (1981) pour la 13/14 et Popescu et Boscher (1982) pour la $4 / 14$. Ces auteurs ont trouvé différents types de caryotypes déséquilibrés dans les embryons âgés de 7 à $10 \mathrm{j}$. Chez les nouveau-nés issus également d'un verrat hétérozygote, ils n'ont trouvé aucun caryotype déséquilibré.

Nous avons effectué une estimation des conséquences économiques d'une translocation réciproque à l'aide d'un modèle de simulation technico-économique (Popescu et Tixier, 1984). Les résultats ont montré que le revenu d'un éleveur de 42 truies utilisant un seul verrat reproducteur hétérozygote pour une translocation réciproque peut être réduit respectivement de 48000 à $32000 \mathrm{~F}$ selon le type de production, soit respectivement de 57 et $48 \%$ du revenu normal (tableau I).

Chez les autres espèces domestiques, tel le cheval ou le lapin, on connaît très peu ou pas d'anomalies de structure en raison, sans doute, d'un échantillon très réduit d'animaux étudiés.

\section{CONCLUSION}

L'ensemble des faits révèle que d'une façon générale les anomalies de la structure chromosomique ne permettent pas une évolution normale de la fonction de reproduction des animaux d'élevage. Elles réduisent deux caractères zootechniques importants : la fertilité et la prolificité. En raison de leurs conséquences économiques néfastes, leur étude constitue un des

Fig 4. Embryon bovin mâle monosomique pour le chromosome 1. 


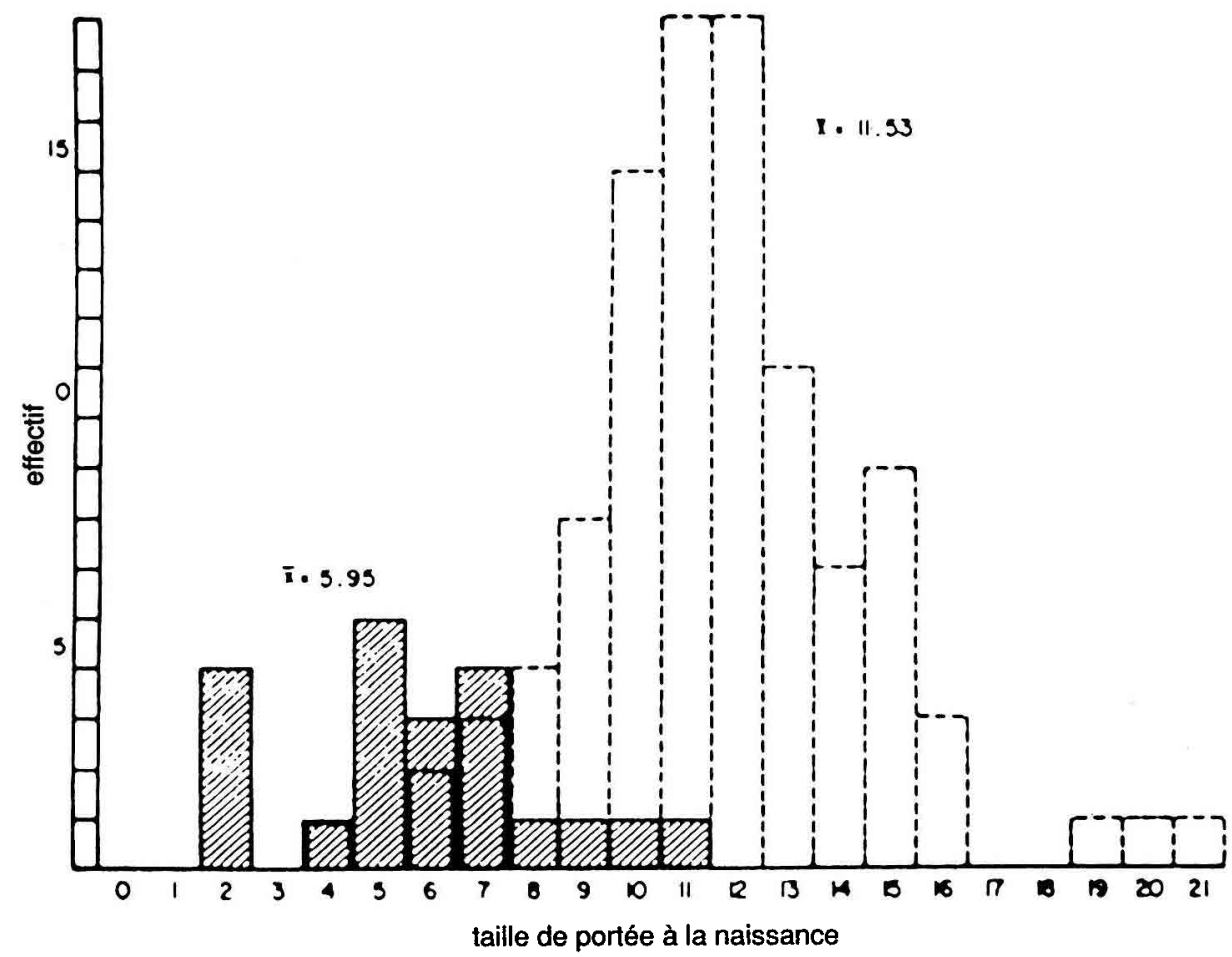

Fig 5. Histogrammes de la taille des 21 portées conçues par un verrat porteur de la translocation 4/14 (en hachuré) et des 95 portées conçues par les verrats contemporains du même troupeau (en pointille). Taille correspondante des portées : $5,95 \pm 2,39$ et 11,53 $\pm 2,91$.

Tableau I. Evaluation du coût de l'utilisation d'un verrat porteur d'une translocation réciproque dans un élevage de 42 truies du $1^{\text {er }}$ au $3^{\ominus}$ trimestre 1982 (Popescu et Tixier, 1984).

\begin{tabular}{lcc} 
Coût total & 32781 & 48332 \\
$\begin{array}{l}\text { Période de réduction } \\
\text { du revenu } \\
\text { (trimestre) }\end{array}$ & $\begin{array}{c}2-82 \\
\text { à }\end{array}$ & $\begin{array}{c}2-82 \\
1-83\end{array}$ \\
$\begin{array}{l}\text { Coût relatif au revenu total } \\
\text { d'un éleveur normal } \\
\text { sur la même période }\end{array}$ & $57 \%$ & $3-83$ \\
\hline
\end{tabular}




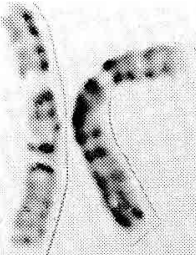

1

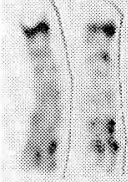

6

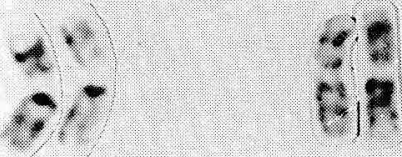

8

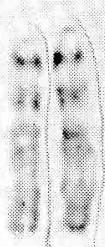

13

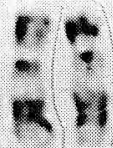

2

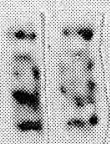

7

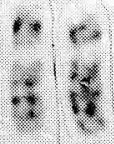

3

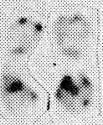

10

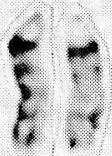

15

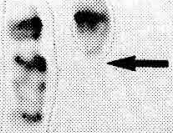

4. 49

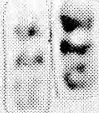

5

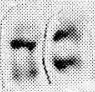

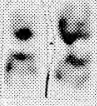

11

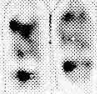

16
12

$x \times$
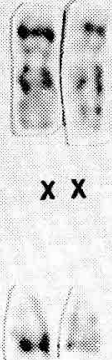

17

Fig 6. Caryotype d'une truie porteuse de la translocation 4/14.

domaines les plus importants de la cytogénétique des animaux domestiques.

\section{RÉFÉRENCES}

Akesson A, Henricson B (1972) Embryonic death in pigs caused by unbalanced karyotype. Acta Vet Scand 13, 151-160

Boué J, Boué A (1975) Etude prospective des facteurs chromosomiques de qualité des gamètes. Ann Biol Anim Biochim Biophys 15, 757-763
Bruere AN (1974) The segregation patterns and fertility of sheep heterozygous and homozygous for three different robertsonian translocations. J Reprod Fertil 41, 453-464

Bruere AN, Chapman HM, Jaine PM (1976) Origin and significance of centric fusions in domestic sheep. J Hered 67, 149-154

Chandley AC (1981) The origin of chromosomal aberrations in man and their potential for survival and reproduction in the adult human population. Ann Génét 24, 5-11

Eldridge FR (1975) High frequency of a robertsonian translocation in a herd of British White cattle. Vet Rec 96, 71-72 
Frebling J, Foulley JL, Berland HM, Popescu CP, Cribiu EP, Darre R (1987) Résultats de l'enquête sur la fréquence de la translocation 1/29 en race bovine Blonde d'Aquitaine. Bull Tech CRZV INRA 67, 49-58

Gustavsson I (1969) Cytogenetics distribution and phenotypic effects of a translocation in Swedish cattle. Hereditas 63, 68-169

Gustavsson I, Rockborn G (1964) Chromosome abnormality in three cases of lymphatic leukaemia in cattle. Nature 203, 990

Hari JJ, Franceschi P, Casabianca F, Boscher J, Popescu CP (1984) Etude cytogénétique d'une population de bovins Corses. CR Acad Agric Fr 70, 191-199

King WA, Linares T, Gustavsson I, Bane A (1980) Presumptive translocation type trisomy in embryos sired by bulls heterozygous for the 1/29 translocation. Hereditas 92, 167169

King WA, Gustavsson I, Popescu CP, Linares T (1981) Genetic products transmitted by rcp $(13 q-; 14 q+)$ translocation heterozygous pigs, and resulting embryonic loss. Hereditas 95, 239-246

Popescu CP (1972) Mode de transmission d'une fusion centrique dans la descendance d'un bouc (Capra hircus L) hétérozygote. Ann Génét Sél Anim 4, 355-361

Popescu CP (1980) Cytogenetics study on embryos sired by a bull carrier of $1 / 29$ translocation. 4th European Colloquium on Cytogenetics of Domestic Animals, Uppsala, 182-186

Popescu CP (1989) Cytogénétique des Mammifères d'Elevage. INRA, 114 pages

Popescu CP, Boscher J (1982) Cytogenetics of preimplantation embryos produced by pigs heterozygous for the reciprocal translocation 4q+;14q-. Cytogenet Cell Genet 34, 119-123

Popescu CP, Tixier M (1984) L'incidence des anomalies chromosomiques chez les animaux de ferme et leurs conséquences économiques. Ann Génét 27, 69-72

Popescu CP, Legault C (1988) Anomalies chromosomiques et "hypoprolificité" chez le porc. $20^{\ominus}$ Journée de la Recherche Porcine en France, Paris, 2-4 février 1988, ITP, Paris, 297-304

Ricordeau G (1972) Observations sur les caractères de reproduction des produits mâles et femelles issus d'un bouc porteur d'une "fusion centrique". Ann Génét Sél Anim 4, 593-598 